\title{
Effects of an External Perturbation on a Cylindrical Spheromak
}

\author{
M. R. Terry
}

May 13, 2002

U.S. Department of Energy

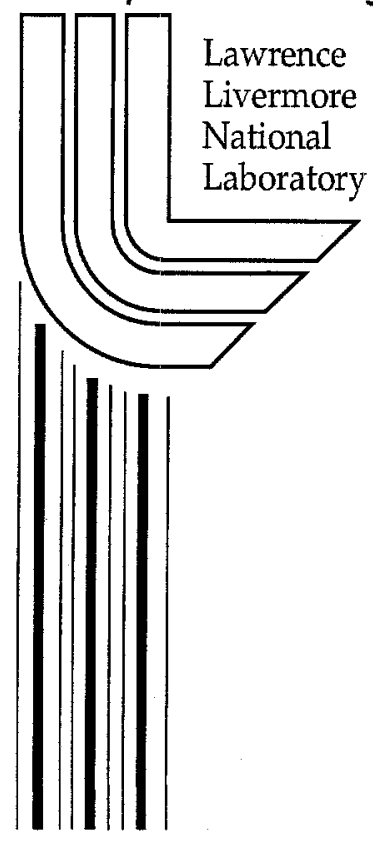




\section{DISCLAIMER}

This document was prepared as an account of work sponsored by an agency of the United States Government. Neither the United States Government nor the University of California nor any of their employees, makes any warranty, express or implied, or assumes any legal liability or responsibility for the accuracy, completeness, or usefulness of any information, apparatus, product, or process disclosed, or represents that its use would not infringe privately owned rights. Reference herein to any specific commercial product, process, or service by trade name, trademark, manufacturer, or otherwise, does not necessarily constitute or imply its endorsement, recommendation, or favoring by the United States Government or the University of California. The views and opinions of authors expressed herein do not necessarily state or reflect those of the United States Government or the University of California, and shall not be used for advertising or product endorsement purposes.

This work was performed under the auspices of the U.S. Department of Energy by the University of California, Lawrence Livermore National Laboratory under Contract No. W-7405-Eng-48.

This report has been reproduced directly from the best available copy.

Available electronically at http://www.doc.gov/bridge

Available for a processing fee to U.S. Department of Energy And its contractors in paper from

U.S. Department of Energy

Office of Scientific and Technical Information

P.O. Box 62

Oak Ridge, TN 37831-0062

Telephone: (865) 576-8401

Facsimile: (865) 576-5728

E-mail: reports@adonis.osti.gov

Available for the sale to the public from

U.S. Department of Commerce

National Technical Information Service

5285 Port Royal Road

Springfield, VA 22161

Telephone: (800) 553-6847

Facsimile: (703) 605-6900

E-mail: orders@ntis.fedworld.gov

Online ordering: http://www.ntis.gov/ordering.htm

\section{OR}

Lawrence Livermore National Laboratory

Technical Information Department's Digital Library

http://www.llnl.gov/tid/Library.html 
Effects of an External Perturbation on a Cylindrical Spheromak

\author{
M.R. Terry \\ Lawrence Livermore National Laboratory \\ Livermore, CA 94551 \\ May 13 - August 2, 2002
}




In experiments like the spheromak, it becomes interesting to investigate the quality of the magnetic flux surfaces in the device. One method of doing so is to impose an external perturbation.

If the magnetic field without perturbation is tangled and no flux surfaces exist, then the perturbation will have little effect. However, if the field has well defined flux surfaces, the field should react strongly to a perturbation. Magnetic islands should form and potentially cause a degredation of the plasma

This paper will assume the second case of good behavior and closed surfaces and will attempt to describe the effects of an external perturbation.

Geometry-wise, we begin with the infinite cylinder approximation to a toroidally confined plasma. In the unperturbed state of this cylinder there are a toroidal field (axial field in the cylindrical geometry), a poloidal field and no radial field. The axial field is assumed to be constant and the poloidal field is described by:

$$
B_{y}=\frac{2 \pi B_{z} r}{q(r) L}
$$

where $B_{\text {theta }}$ is the poloidal field, $B_{z}$ is the toroidal field, $r$ is the distance from the geometric axis, $q$ is the safety factor, $I$ is the length of one period of the cylinder.

We assume that the safety factor can be described by:

$$
\frac{1}{q}=\frac{1}{q_{a}}-r\left(\frac{1}{q_{a}}-\frac{1}{q_{e}}\right)
$$

Where $q_{a}$ is the value of the safety factor at the axis of the cylinder and $\mathrm{q}_{e}$ is the value at the edge.

The values of $q_{a}$ and $q_{e}$ were chosen to be feasible values for the LLNL SSPX spheromak and to highlight situations of interest.

We began making our magnetic field line tracing by assuming that given an initial point ( $r$, theta, $z$ ) within the cylinder on $a$ magnetic field line, another point on the same field line could be found at $(r+$ deltar, theta + deltatheta, $z+$ deltaz)

where

$$
\left[\begin{array}{l}
r_{i+1} \\
\vartheta_{i+1} \\
z_{i+1}
\end{array}\right]=\left[\begin{array}{l}
r_{i}+\delta r \\
\vartheta_{i}+\delta \vartheta \\
z_{i}+\delta z
\end{array}\right]
$$

$$
\delta \boldsymbol{r}=\frac{\boldsymbol{B}_{\boldsymbol{r}}}{|\boldsymbol{B}|} d s \quad \delta \vartheta=\frac{1}{\boldsymbol{r}} \frac{\boldsymbol{B}_{\vartheta}}{|\boldsymbol{B}|} d s \quad \delta z=\frac{\boldsymbol{B}_{z}}{|\boldsymbol{B}|} d s
$$

As long as ds is chosen to be sufficiently small, this lends itself to an iterative method for tracing the field line. Each point on the magnetic field line can be used to find the next point and so on until you have a full description of the line. 
Going back to the toroidal model, this field line wraps its way around the torus many times both toroidally and poloidally during its lifetime. It becomes interesting to see the shape of this field line at a cross section in the torus. Closed field lines and their accompanying islands as well as other structures in the magnetic field should be readily apparent from this perspective.

In returning to the cylinarical model, this puncture plot is easily generated while tracing the field Iine, Each time the field line travels a distance $L$ along the axis of the cylinder, the code pulis out the ( $r$, theta) coordinates of the field ine and stores these until the trace of the field line is complete.

Imposing a perturbation on the torus is reflected in the cylindrical geometry by placing a series of sources parallel to the cylinder at increments of $\mathrm{I}$. At each point of evaluation, the contribution of each has to be summed for numerically, contributions from successive source must be added until the contribution is smaller than the accuracy of the calculation).

Before embarking on simulating a full external source perturbation on our idealized spheromak, we begin by making inquires into the effect of adding a constant magnitude, single harmonic perturbation.

The magnitude of the radial component of the perturbation is given by:

$$
\operatorname{per}_{r(m, n)}=\left(\epsilon B_{z} r\right) \cos \left(2 \pi \frac{z}{L} m-\vartheta n\right) \quad \operatorname{per}_{\vartheta(m, n)}=\left(\epsilon B_{z} r\right) \cos \left(2 \pi \frac{z}{L} m-\vartheta n\right)
$$

where epsilon is a fraction of $B_{z}$, ( $r$, theta, $z$ ) are the coordinates of the point, $L$ is the period of the cylinder. The harmonic is determined by $m$ and $n$, corresponding to the number of turns toroidally and poloidally, respectively.

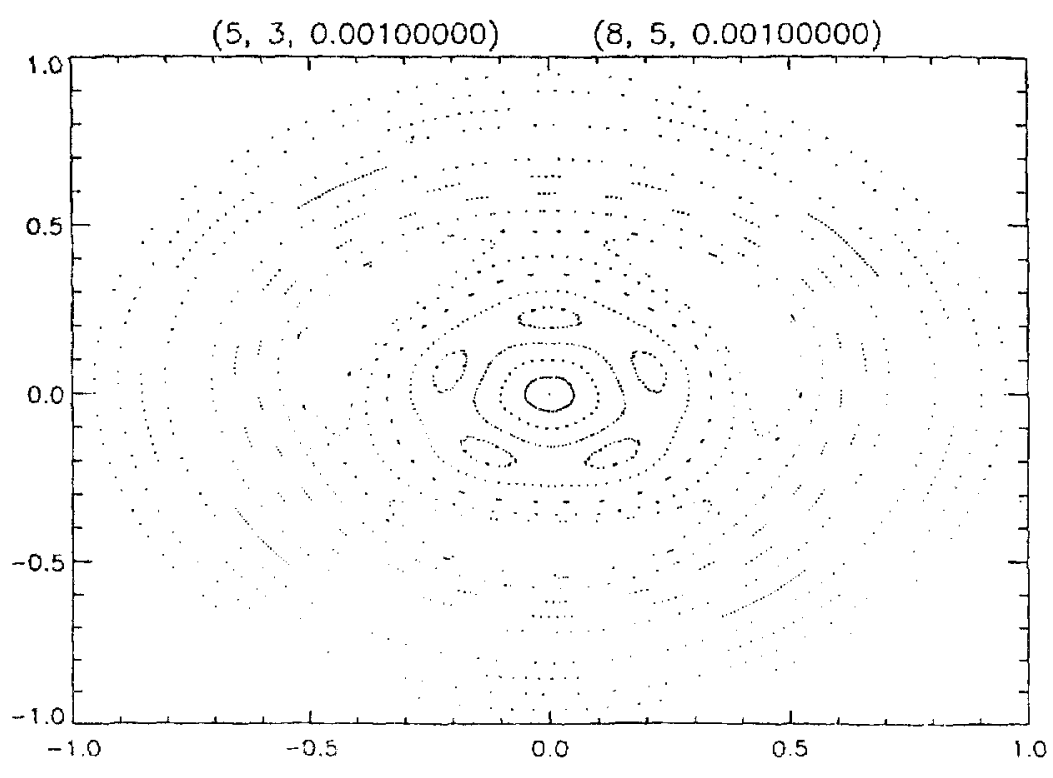

Island formation was distinct and corresponded with the same $q$ surface as was expected (where q $=\mathrm{m} / \mathrm{n})$.
This plot shows two harmonics (at a magnitude of , 18 of $\mathrm{Bz}$ ) and their accompanying islands. It also shows puncture plots for field lines inside, between and outside the magnetic islands. 


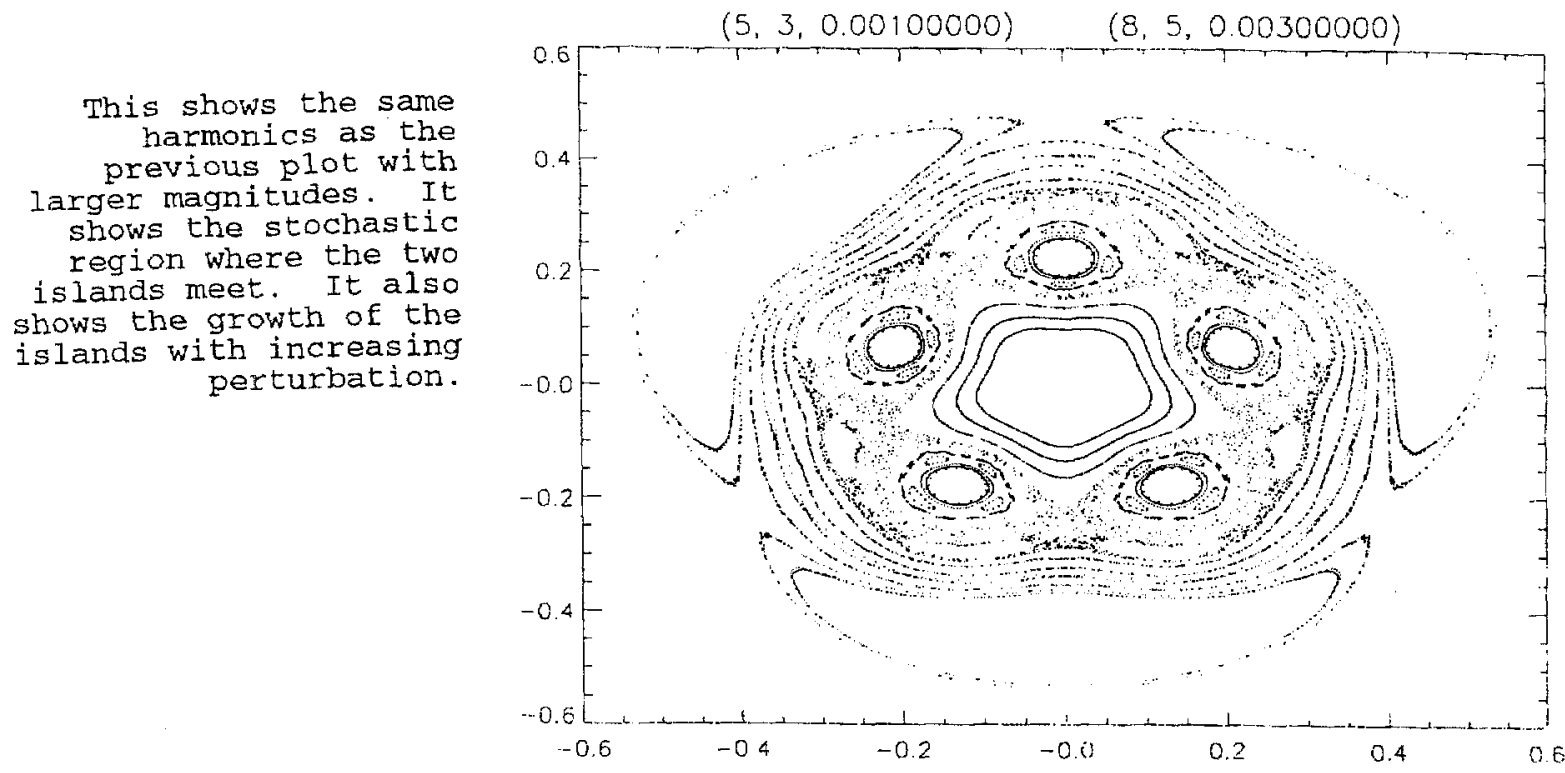

With the addition of a second harmonic, multiple islands located about different flux surfaces were observed. By increasing the perturbation of the respective harmonics, the islands were observed to grow and eventually overlap. Distinct regions of stochasticaliy were observed in the region where the two islands met.

Having shown the effects of imposing a single harmonic and a small number of harmonics, the next step was to illustrate the effects of imposing the full spectrum of harmonics that an external source would impose.

Now that the perturbation varies in space, the placement and orientation matter. Also of significance is that in the cylindrical geometry, imposing a single external source does not model imposing a source on a torus. The periodicity of the torus requires that an array of identical sources be placed along the length of the cylinder at increments equal to the period of the torus. Schematically, the effect of each source must be summed. Numerically, the each effect must be summed until the magnitude of the effect is smaller than the accuracy of the calculation.

The perturbation was initialiy chosen to be a current carrying loop of wire located a distance away from the torus, but the evaluation of an array of eliptic integrals for the magnetic field made the program too slow to be useful. A dipole moment can replace the loop current with only a small

difference in the behavior of the field.

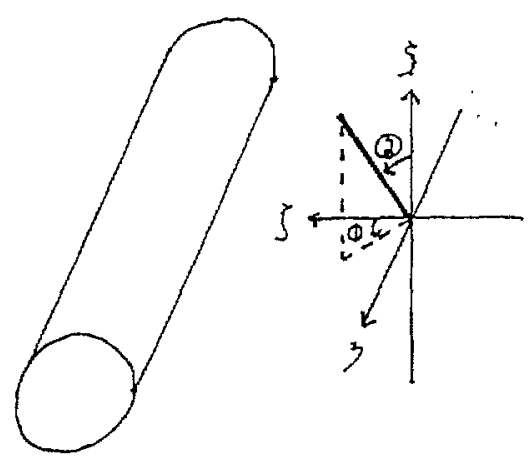


The perturbation due to the dipole situated centrally

$$
\begin{gathered}
\operatorname{per} \boldsymbol{B}=\frac{\mu_{0}}{4 \pi} \frac{3(m \cdot r) r-\left|r^{2}\right| m}{\left|r^{5}\right|} \\
r=\left(d-r \cos (\vartheta), r \sin (\vartheta), z+\left(\alpha-\frac{1}{2}\right) L\right) \quad|m|=\frac{2 \pi \epsilon B_{z} d^{2}}{\mu_{0}}
\end{gathered}
$$

Where $r$ is the vector from the dipole to a point in the cylinder, $m$ is the dipole moment, $d$ is the distance separating the dipole and the cylinder, ( $r$, theta, $z$ ) are coordinates in the cylinder, alpha is an integer representing which dipole in the array it is, $L$ is the period of the cylinder.

The first cases looked into in this configuration were in the vicinity of the $q=1 / 2$ surface. Near and on this surface second order islands would be expected to form. Rather than the island pairs forming as expected, a single crescent formed. The cause for this shape is not totally explained yet. It is likely due to some inaccuracies in the method of making the plots, but this has not been confirmed yet.

The islands formed responded positively to an increase in magnitude of the perturbation.

Island formation was found to be very sensitive to the orientation of the dipole moment. It was found that by skewing the direction of the moment, one could drastically degrade the formation of islands and destroy otherwise circular flux surfaces.

Broadening the range $q$ was found to make the islands more narrow as the radial distance that could be considered "near" $q=1 / 2$ (and where island formation occurs), occupied a smallex fraction of the space.

Shortening the range made the islands thicker as would be expected.

TOP: epsilon $=.01$, aimed directly at cylinder. CENTER: epsilon $=.05$, aimed directly at cylinder, for field line beginning at $r=.8$, theta $=\mathrm{Pi}$

BOTTOM: epsilon $=.05$, aimed $\mathrm{Pi} / 4$ above cylinder, for field ine beginning at $r=.8$, theta $=P i$. Note the chaotic path compared to the previous puncture plot.

SIDENOTE: To give a sense of scale, for a loop current with radius. Im, located $.25 \mathrm{~m}$ away, it takes about $31 \mathrm{kA}$ to have a perturbation 18 of $\mathrm{B}_{z}$ directed inward radially at the cylinder surface nearest the loop.
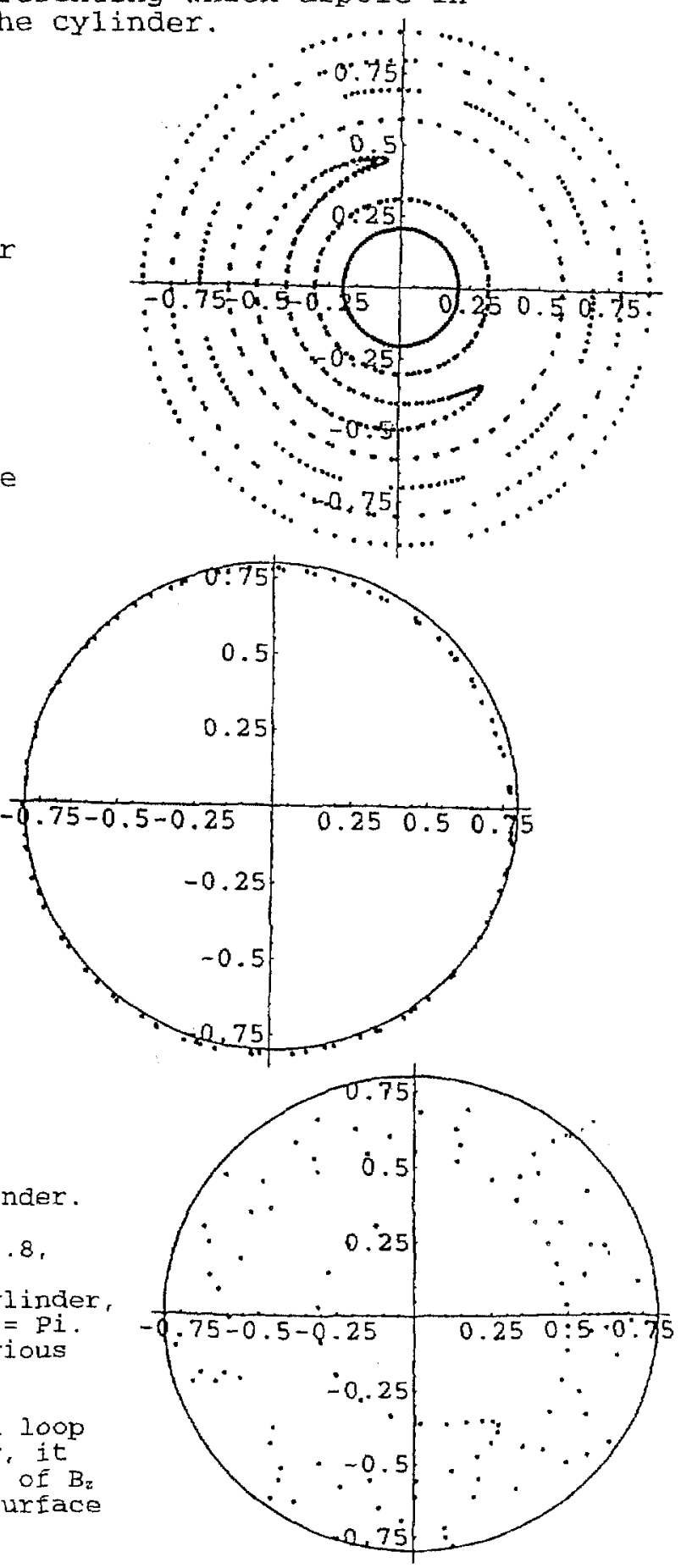
During these runs of the code, issue questioning the small scale accuracy of the code arose. Either a smaller step size was needed to continue using the Euler method or the code should be migrated to a more efficient modified-Euler method or some other method for solving the path of the field line.

Work was begun at making the plasma have a non-cylindrical geometry that more closely resembles the geometry in a spheromak. One way of accomplishing this is by running a current carrying wire above the cylinder. The current is such that there is a magnetic $x$-point at the edge of the plasma. The teardrop shape it plots is confirmed by the analytic expression that describes the system. The agreement between code and analytic expression provide some validation that the mechanism used to extract and plot punctures is valid. The completion of the line current perturbation also puts the code in a stance to begin imposing a perturbation on a spheromak like (non-circular) geometry.



Puncture plot for line current located $208 \mathrm{R}_{\text {minor }}$ above the cylinder. 